\title{
El Cultivo y Crianza de Sabidurías y Conocimientos en el contexto de la comunicación intercultural
}

\author{
The Cultivation and Breeding of Wisdoms and Knowledge in \\ the Intercultural Communication Context
}

Yulmar Runel Montoya Ortega ${ }^{1}$

\section{Resumen}

El Cultivo y Crianza de Sabidurías y Conocimientos (CCRISAC), es una metodología de investigación propia que nace en el seno de la Red de Universidades Indígenas Interculturales y Comunitarias de Abya Yala (RUIICAY), como una propuesta desde los pueblos indígenas y afrodescendientes de Abya Yala. Esta iniciativa se hace enriquecedora desde el ejercicio de la comunicación intercultural, puesto que asegura la relación interpersonal entre el investigador y la comunidad de manera holística, respetuosa y de consenso, es decir, toma como parte esencial y parte de la construcción colectiva de sujeto a sujeto; construye, dialoga, comparte, consensa, valida, socializa y armoniza. La metodología fue el Cultivo y Crianza y Crianza de Sabidurías y Conocimientos, que se visualiza desde la armonización de los conocimientos entre las distintas fuentes bibliográficas. Finalmente, la comunicación intercultural es un elemento indispensable que se materializa desde la relación y correlación entre los sujetos de estudio, es decir, las comunidades son el foco esencial que abren su mente al investigador para poder construir comunitaria e interculturalmente. Por ello, todo investigador e investigadora, debe de abrir su mente al ejercicio de una comunicación diferente, humana y que se hace visible desde el entendimiento respetuoso entre diferentes.

Palabras Claves: CCRISAC; Comunicación Intercultural; Investigación Propia.

\section{Abstract}

The Cultivation and Breeding of Wisdoms and Knowledge (CCRISAC, for its translation into Spanish) is a methodology of own research that is born within the Network of Indigenous Intercultural and Community Universities of Abya Yala (RUIICAY), as a proposal from the indigenous and afro-descendant peoples of Abya Yala. This initiative is enriching from the exercise of intercultural communication, since it ensures the interpersonal relationship between the researcher and the community in a holistic, respectful and consensus way, that is, it takes as an essential part and begins from the collective construction of subject to subject; builds, dialogues, shares, consents, validates, socializes and harmonizes. The methodology was the Cultivation and

1 Máster Internacional en Comunicación Intercultural con enfoque de género. Director del Instituto para la Comunicación Intercultural de la Universidad de las Regiones Autónomas de la Costa Caribe Nicaragüense (URACCAN). Correo: yulmar.montoya@uraccan. edu.ni; No. ORCID: https://orcid.org/oooo-0003-1873-2026

Recibido: 14/12/2018 Aprobado: 31/05/2019 
Breeding of Wisdoms and Knowledge, which is visualized from the harmonization of knowledge between the different bibliographical sources. Finally, intercultural communication is an essential element that materializes from the relationship and correlation between the subjects of study, that is, communities are the essential focus that open their minds to the researcher to be able to build community and interculturally. Therefore, every researcher must open his or her mind to the exercise of a different, human communication that becomes visible from the respectful understanding among the differences.

Keywords: CCRISAC, Intercultural Communication, Own Investigat.

\section{Introducción}

Los procesos de investigación se realizan mediante diferentes metodologías, según el tipo de estudio y el procedimiento que se establece para desarrollar cualquier temática que se pretende desarrollar. Obviamente, estos procedimientos parten de una necesidad y de un objetivo común. Mismo que pretenden demostrar o encontrar algún resultado de interés. Entre los estudios más habituales se encuentran los de carácter cuantitativo y cualitativo. El enfoque de investigación cuantitativa obedece a patrones comunes que muchas veces excluyen el sujeto de estudio. Por lo que el investigador o investigadora, comparte a su universo el interés de realizar dicho trabajo hasta cuando ya está listo para realizar y aplicar instrumentos. Generando así un proceso no adecuado para la realización del mismo, considerando que en el caso de las comunidades y pueblos indígenas y afrodescendientes son vistos como objetos de estudio.

De tal manera que, entre las principales características del método cualitativo, resalta que se debe de tener en cuenta, con carácter primordial, el objeto de la investigación. Si una investigación pretende captar el significado de las cosas (procesos, comportamientos, actos), más bien que describir los hechos sociales, se puede decir que entra en el ámbito de la investigación cualitativa, (Olabuénaga, 199). De modo que este ejercicio y por su naturaleza de trato, transgrede la libre determinación de quienes son parte de una iniciativa, que más allá de querer aportar a mejorar condiciones en el caminar, excluyen la construcción colectiva. La consulta previa es un derecho fundamental del que son titulares los grupos étnicos, Charris (2014), pueblos Indígenas y Afrodescendientes.

Es así que, se debe estar claro de la necesidad de crear espacios, caminos y formas que permitan garantizar la creación de elementos colectivos que apunten al Buen Vivir, desde la lógica colectiva y vivencial del sujeto de estudio. Verlo e interpretarlo como tal. Ante este tipo de cuestiones, es indisoluble fomentar las buenas prácticas, creando estrategias, métodos propios para realizar una investigación que apunte a la armonía y a la construcción plural. Por ello, es importante considerar y retomar las 
buenas prácticas e iniciativas implementadas para ir dando respuesta a este tipo de problemáticas y cuestionamientos.

Desdela Red de Universidades Indígenas Interculturales y Comunitarias de Abya Yala ${ }^{2}$ (RUIICAY), se han venido realizando esfuerzos colectivos que apuntan a la construcción de una propuesta metodológica de investigación propia, que aporte al Buen Vivir y al Vivir Bien de los pueblos indígenas y afrodescendientes. Mediante un tratamiento integrador, consenso y diálogo de saberes colectivos, se construyó la propuesta del Cultivo y Crianzas de Sabidurías y Conocimientos, CCRISAC.

Es así que esta propuesta metodológica parte de procesos colectivos desde y para los pueblos, nacionalidades y culturas, para fortalecer y revitalizar su autonomía, autodeterminación, identidad, sabidurías, expresiones, conocimientos, prácticas y episteme (RUIICAY; 2015).

En este sentido es necesario entender cómo se puede materializar el Cultivo y Crianza de Sabidurías y Conocimientos, en el ejercicio de la comunicación intercultural, para un ejercicio más enriquecedor. De tal manera que el presente artículo, pretende describir los elementos fundamentales del CCRISAC y el papel de la comunicación intercultural mediante su aplicación.

\section{Materiales y métodos}

La identificación de autores que resaltan y abordan esta temática, jugaron un papel clave para el abordaje y construcción del presente escrito, porque permitió socializar, debatir y reforzar las dinámicas compartidas durante el desarrollo de todo el proceso descrito en este contenido. Se realizó una revisión documental exhaustiva para dar mayor fuerza y valor al artículo y se consultaron documentos escritos por Alta Hooker, Asunción Lande, Jorge Velásquez, Neydi Calderón, Miquel Alsina, Hilvert Timmer, autores especialistas en educación y comunicación intercultural, quienes además de escribir sobre comunicación intercultural también aportan a la conceptualización. Pero también se revisó documentos institucionales de la RUIICAY y URACCAN, quienes escriben sobre este tipo de contenidos desde el ejercicio colectivo fundamentado en la interculturalidad. En la búsqueda de la literatura se incluyó temática referidas a la comunicación intercultural desde la construcción de tesis de investigación inéditas sobre la temática en mención en el marco de la Maestría Internacional en Comunicación Intercultural con enfoque de género, la interculturalidad, la metodología de investigación propia (CCRISAC), la planificación estratégica de URACCAN, el papel de la comunicación en procesos de interculturalidad, la chakana, la comunicación interculturalidad y la comunicación intercultural, que se visualizan desde sus escritos, pero también en relación con otros autores.

2 Abya Yala: es el nombre propio que hace referencia a América Latina, dado por el pueblo indígena Kuna. 


\section{Criterios de inclusión y exclusión}

En la revisión bibliográfica se incluyó escritos elaborados por cada uno de los autores, como un aporte a la construcción del presente escrito que hacen un análisis crítico y profundo sobre estos procesos que aún están en construcción, pero que cada vez se materializan desde las diferentes vivencias y experiencas presentadas. Sobre las revisiones sistemáticas y los estudios científicos se aplicó como criterio de inclusión que los escritos abordan aspectos sustanciales sobre la comunicación intercultural, el Cultivo y Crianza de Sabidurías y Conocimientos (CCRISAC), y la creación, recreación de conocimientos, saberes y prácticas. El único criterio de exclusión fue que los artículos no incluyeran información sobre la temática en cuestión.

\section{Fuentes de datos}

De un total de 30 escritos con autores, se extrajeron artículos que aportan a la temática desarrollada en este artículo. Para proceder a la selección se revisaron los artículos completos con el fin de analizar, criticar y decidir por la información necesaria y que estaba relacionada con el objetivo de este escrito.

\section{Análisis de los datos}

La información analizada se estructuró en tres espacios: El papel de la comunicación intercultural en el CCRISAC, características de la investigación propia y el fruto de la comunicación intercultural, Caminos del CCRISAC, su implementación y conclusiones. Finalmente, toda la información extraída, se revisó sobre autoría, año, finalidad, fuentes de información.

De los artículos originales se obtuvo información sobre autoría, libros, tesis y revista en la que han sido publicados y año de publicación, país donde se realizó el estudio, tipo de estudio, sujetos, origen y conclusiones.

\section{Resultados y discusión}

\section{El papel de la comunicación intercultural en el CCRISAC}

La comunicación intercultural es un elemento fundamental que permite la vinculación directa entre el investigador y la comunidad, porque se materializa de manera horizontal, de sujeto a sujeto y permite el entendimiento, el consenso y la reciprocidad para la construcción colectiva de propuestas que trazan un camino para responder a necesidades o demandas de los involucrados, buscando un bien común para el Buen Vivir y el Vivir Bien. Podemos hablar de comunicación intercultural como la comunicación entre aquellas personas que poseen unos referentes culturales tan distintos que se auto perciben como pertenecientes a culturas diferentes, Alsina (1999). Es 
por ello que no podemos hablar del CCRISAC sin antes hacer hincapié en la comunicación; puesto que en gran escala esta metodología de investigación propia, se hace real desde la fundamentación de una idea hasta el desarrollo e implementación de sus resultados, como una forma de cumplir con criterios que determinan el compromiso adquirido por quien convive y realiza el trabajo investigativo. El papel fundamental de la comunicación intercultural parte de la espiritualidad y de construir puentes de confianza (Hooker, 2017, p. 15).

El papel de la comunicación intercultural fortalece el aprender relaciones entre diferentes, abre el camino para la relación interpersonal y con los demás seres, y por ende los procesos encaminados desde cada una de las cosmovisiones propias de cada pueblo de cara al Buen Vivir y el Vivir Bien.

La comunicación intercultural en este contexto, es un elemento vivo que se vincula de inicio a fin, porque hay un trecho relacional directo e indirecto con la comunidad, institución u organización que es parte del proceso. Esta vinculación se describe en el respeto, unidad, consenso y validación. De manera que es esencial partir de que la comunicación, es un espacio abierto y permanente que aporta a la consolidación de los espacios en tiempos de siembras y cosechas. Que como espacio del compartir la palabra retorna a la comunidad para su implementación. Pero además los miembros sujetos, juegan un papel determinante porque son quienes abren sus mentes para compartir los conocimientos saberes y prácticas desde su cosmovisión, desde el compromiso y colaboración. La comunicación intercultural puede ayudar a crear una atmósfera que promueva la cooperación y el entendimiento entre las diferentes culturas, y posee características especiales que le pueden permitir realizar tal función, Lande (1986).

Desde esta perspectiva, la comunicación intercultural, mantiene una vinculación permanente entre los sujetos y que se hace real a través del diálogo de saberes y haceres, mediante la vivencia, el bien hacer y el materializar la interculturalidad desde la relación misma, que vuelve natural el intercambio de conocimientos comunitarios, interculturales, inter científicos e inter epistémicos a través del CCRISAC, como una propuesta nueva y diferente de metodología de investigación. El CCRISAC, permite vivenciar, convivir, recrear, armonizar y fortalecer los conocimientos saberes y prácticas desde el diálogo de saberes y haceres, mismos que constituyen una fortaleza fundamental para el Bien Hacer y el Buen Vivir de los Pueblos, (RUIICAY, 2018).

Por ello, es vital y fundamental el desarrollo de la comunicación en todas las etapas del desarrollo del Cultivo y Crianza de Sabidurías y Conocimientos (CCRISAC), metodología propia de investigación que incluye y prioriza la comunidad poniendo de inter medio el diálogo intercultural. Además, esta propuesta garantiza la armonía y la resiliencia con espacios de articulación sinérgicos mediados por los actores claves que forman parte del proceso constructivo del proceso investigativo en todos los 
niveles. Sin embargo, para implementar dicho proceso se debe empezar un diálogo intercultural para conocer a los otros, Alsina (1997).

\section{Características de la investigación y la comunicación intercultural}

El CCRISAC es una propuesta en construcción y que se materializa desde el sentir de los Pueblos Indígenas y Afrodescendientes en la búsqueda de la creación de un sistema inclusivo que los vuelva parte e involucre en la Creación y Recreación de Conocimientos Saberes y Prácticas, partiendo de sus realidades y contextos, que abona a la construcción de ciudadanías interculturales. Esta iniciativa es fundamental y estratégica porque aporta a las demandas históricas de los pueblos por ser sujetos y no objetos de estudios. A su vez, es una manera de ver y entender el mundo desde una visión holística y de derechos.

El CCRISAC, se concibe como una propuesta pedagógica y metodológica con posicionamiento epistémico y filosófico para el desarrollo de los procesos de Creación, Recreación de Conocimientos, Saberes y Prácticas de los Pueblos, las organizaciones e instituciones, como un aporte de la RUIICAY. Aporta a la construcción de procesos investigativos de una manera holística, donde las comunidades sean sujetos y no objetos de estudios (RUIICAY, 2018, p. 9).

El Cultivo y Crianza de Sabidurías y Conocimientos (CCRISAC), destaca características fundamentales que se desprenden de su implementación como una filosofía de vida. Por lo tanto, quienes desde los pueblos que integran la RUIICAY han compartido sus posicionamientos epistémicos en esta construcción colectiva que aporta a un ejercicio científico diferente. Hemos depositado la semilla de las sabidurías y conocimientos de los pueblos de Abya Yala en este documento, mediante un diálogo respetuoso y profundo, donde hemos sido hebras y nudos de este tejido continental, (RUIICAY, 2018, p. 4). Teniendo en cuenta este punto de partida, desde mi criterio comparto que esta metodología de investigación propia se materializa mediante las siguientes características.

Inclusiva y de consenso: Porque involucra directamente a la comunidad y construye desde el sentir de los pueblos. Su punto de partida es el consenso que se materializa en el entendimiento entre sujetos. Es decir, el investigador o investigadora va a la comunidad presenta su propuesta y realiza el proceso de Consulta Previa Libre e Informada, que responde a la autodeterminación de los pueblos indígenas y afrodescendientes como un derecho fundamental.

Armoniza y articula: Armoniza los conocimientos tradicionales con los denominados científicos, ejercicio que permite fortalecer los procesos investigativos puesto que articula el conocimiento endógeno con el exógeno; dándole mayor vistosidad y 
rigurosidad al proceso constructivo con la revisión documental y el levantamiento de la información con las fuentes vivas.

Valida y convive: Su mayor fortaleza se materializa en la validación de la información, que se teje a través de la convivencia y el compartir la palabra mediante distintas formas de convivencia: asambleas comunitarias, danza, arte, alimentos, espiritualidad, rituales y trabajo comunitario.

Dialoga y construye: A través de los actores claves se realizan espacios de diálogos, que garantiza la ruta de inicio a fin incluida la implementación de la hoja de ruta que el investigador o investigadora determine con los miembros de la comunidad. Pero además se construye la propuesta de manera conjunta para luego continuar con el proceso constructivo desde los caminos y formas que establece el CCRISAC.

La palabra es vida y sueño: La mayor fortaleza de los pueblos y sus conocimientos se transmiten a través de la palabra, la oralidad es un factor fundamental y determinante para su cosmovisión, porque el conocimiento ancestral es herencia sagrada, pues desde el caminar la palabra fortalecen y tejen la vida, sus conocimientos, cultura, espiritualidad, que la comparten desde los sueños y anhelos de sus territorios, que consiste en el cuido y protección de los recursos de la madre tierra, porque conviven y hablan con la madre naturaleza, dialogan, comparten y piden permiso a la naturaleza.

Aprehende y desaprehende: Es un desafío congruente con el pensamiento ancestral, que se materializa a través de la cosmovisión de cada pueblo y se arraiga desde el conocimiento tradicional. Parte de la mente abierta que reconoce el conocimiento tradicional y que busca entenderlo y conocerlo. Para inmiscuirse en todo el proceso aprende lo que en su mente puede ser nuevo y desaprende lo que occidente o la ciencia establece como verdad absoluta.

Teje y colorea: El tejido es la fórmula por excelencia desde el sentir cosmogónico del pensamiento indígena y afrodescendiente, pues ese ejercicio les permite proyectar, armar y plasmar desde la razón del corazón el sentir y la significación de cada cultura. Es la acción fundamental que marca el caminar y la ruta de sujeto a sujeto para colorear el caminar de la palabra y el camino mostrado por los ancestros.

Para implementar y hacer visible esta metodología de investigación, es necesario una comunicación humana, fluida y de compromisos; aquí entra estratégicamente la comunicación intercultural que desde sus virtudes y bondades rompe paradigmas y crea espacios de entendimiento y de reciprocidad a través del compartir la palabra. La comunicación, es uno de los principales elementos que dan vida a cualquier raza viva, desde sus particularidades lingüísticas que predominen según sus realidades y cultura, (Calderón, 2018, p. 40). De esa relación estratégica que el investigador y la comunidad mantienen y que construyen a través del consenso. Pero que además retorna 
con los nuevos conocimientos adquiridos para implementar de manera respetuosa y consensuada entre los involucrados.

No cabe duda que la construcción de la investigación pasa primero por la comunicación intercultural porque es el puente de negociación, de diálogo y de consenso entre las partes, que acuerdan seguir un camino común que responde a las necesidades, demandas y planes de vida de sus comunidades. Y entiéndase por interculturalidad la forma de interacción entre las culturasdiferentes, de ahí que la comunicación intercultural garantiza el diálogo horizontal y franco.

La interculturalidad, por tanto, es interpretada como una interacción entre culturas donde los procesos de comunicación juegan un papel preponderante, por lo que se intuye entonces, la necesidad de una comunicación que garantice ese dialogo horizontal, de ahí la necesidad de la comunicación Intercultural (Velásquez; 2018, p. 15).

Esta propicia el encuentro, la relación armónica de sujeto a sujeto que se hace visible por medio del diálogo intercultural y de la relación directa que mantiene el investigador con la comunidad. Estos procesos son posibles gracias a la comunicación intercultural, que se vincula desde la gestión de la misma para hacer realidad un proceso constructivo pluricultural.

La comunicación Intercultural, debe significar oportunidades para acercarnos desde nuestros espacios a demandas sugeridas y propias de la comunidad y la sociedad en su conjunto, en un esfuerzo por lograr que quien recibe información, pueda aportar desde su condición de sujeto y no objeto receptor, en el que prevalezca el respeto a su identidad cultural, territorial, espiritual y económica, sin transgredir el ser indígena, el ser negro o el ser campesino, sino que haya un abordaje adecuado y lógico, según las particularidades y demandas de los pueblos (Montoya, 2018, p. 34).

\section{Caminos del CCRISAC}

Los Caminos del CCRISAC son los que occidente entiende como metodologías de investigación, que se generan en tres procesos diferenciados fundamentados desde cada una de las universidades que han sido parte de la construcción de este proceso, es el caso de la Universidad Autónoma Indígena Intercultural (UAIIN) del Consejo Regional Indígena del Cauca (CRIC), Colombia; de la Pluriversidad Amawtay Wasi del Ecuador; y de la Universidad de las Regiones Autónomas de la Costa Caribe Nicaragüense (URACCAN), respectivamente. Estas tres casas del conocimiento mayor han sido las fundadoras de la RUIICAY, han construido procesos a través del diálogo horizontal y armonioso, haciendo realidad la práctica de la comunicación intercultural. Este documento presenta los caminos del Cultivo y Crianza de Sabidurías y Conocimientos de algunas universidades miembros de la RUIICAY, (RUIICAY, 2018, p. 9). En esta 
ocasión describiré cada uno de los caminos, pero haré más hincapié en el Camino de Creación y Recreación de Conocimientos Saberes y Prácticas.

La Chakana: Es la metodología propuesta por la Universidad Autónoma Indígena Intercultural (UAIIN), Colombia, que recoge la cosmovisión del mundo andino, desde la llamada ancestralmente Cruz del Sur, que se visiona en gran escala en los países de sur América, particularmente en toda la franja de los pueblos andinos. Y, tiene una relación con el cosmos y los espacios espirituales, los tiempos y las cosechas. Es sagrada para los pueblos indígenas de Abya Yala.

La Chakana hace referencia a la constelación "Cruz del Sur”, visible en nuestro territorio. Ha sido muy utilizada por los pueblos originarios como el relacionamiento con el cosmos en las construcciones, caminos, orientaciones y ritualidades. Un primer mensaje de la Chakana se refiere a la ética de quién indaga. Proviene de su relación armónica con la Pachamama (Madre Tierra), ejemplo, al ser Runa Yanakuna es una persona integral o una persona articulada con el territorio (RUIICAY; 2018, p. 13).

Timmer (2003; p. 1), detalla el punto de entendimiento y aspectos sustanciales sobre la existencia e importancia que tiene la chakana para los pueblos indígenas andinos que tiene un significado importante en el universo simbólico:

La cruz ya existe desde hace 1000 años y funcionaba para los ancestros como adivino de la rotación de la Tierra y era la indicación para las épocas de la siembra y de la cosecha, para la lluvia, heladas, el granizo y la nieve. Además de ser un supuesto calendario, era un símbolo geométrico y matemático que podría poner orden. Por regla general, se atribuye el origen de la chakana a la constelación de las estrellas que se puede ver en el hemisferio austral y que se conoce por la Cruz del Sur.

Vivencial simbólico relacional: Esta propuesta fundamentada por la Pluriversidad Amawtay Wasi del Ecuador, consiste en construir desde la vivencia, desde la relación directa entre las comunidades, que son la clave fundamental para la construcción y cosecha de sus semillas. Además, se fundamenta en la complementariedad y entiende como un principio la convivencia, el compartir, la reciprocidad, la relación horizontal.

Este método recupera principalmente el sentido de par complementario en la práctica y la teoría. el método vivencial-simbólico-relacional, conmina a aprender vivenciando, viviendo plenamente los hechos, conjugando la razón con la intuición, lo vivido con lo que se quiere vivir, en estrecha relación entre lo individual y lo comunitario, entre lo material y lo espiritual, (RUIICAY; 2018, p. 25). 
Creación y recreación de conocimientos, saberes y prácticas: Este camino, promueve el buscar nuevos conocimientos, crear aspectos sustanciales en materia de investigación, pero también recrear conocimientos, fortalece los saberes y lo materializa desde la práctica en la implementación de las semillas que germinan desde el proceso constructivo que involucra a los sectores involucrados en el Cultivo y Crianza de Sabidurías y Conocimientos (CCRISAC). Pero además armoniza, convive y construye en colectividad.

Se define como un proceso participativo, que genera nuevos conocimientos, capacidades e innovaciones. Sistematiza y revaloriza las prácticas históricas y saberes locales de los pueblos indígenas, afrodescendientes, mestizos y comunidades étnicas en la búsqueda del Buen Vivir (URACCAN, 2014, p. 22).

Esta propuesta se desarrolla a través de varios momentos, que son las líneas que te permiten caminar paso a paso e ir construyendo tu cultivo y crianza de manera holística, ordenada y sigilosamente te permite pensar y repensar la ruta que deseas trazar de manera colectiva, es decir, entre el investigador y los sujetos de estudios. Esta se fundamenta y nace desde el sentir de los pueblos indígenas y afrodescendientes de la Costa Caribe Nicaragüense a través de la Universidad de las Regiones Autónomas de la Costa Caribe Nicaragüense (URACCAN) "Primera Universidad Comunitaria Intercultural en América Latina".

En este proceso de construcción y fortalecimiento del modelo de Universidad Comunitaria Intercultural, la URACCAN ha venido construyendo una serie de principios y conceptos institucionales que buscan transcender hacia un modelo propio que promueve el diálogo epistemológico, donde los saberes, conocimientos y prácticas de los pueblos indígenas, afrodescendientes, mestizas y otras comunidades étnicas, ocupen un lugar protagónico en la búsqueda de Autonomías, el Buen Vivir, construcción de ciudadanías interculturales de género para un Desarrollo con Identidad (RUIICAY, 2018, p. 29).

Esta propuesta se materializa a través de varios momentos que describiré a continuación desde mi entender en complementariedad con la estructuración de los momentos que establece del CCRISAC (RUIICAY, 2018):

\section{Primer momento: Soñar la Idea}

Parte del consenso, es decir, se presenta una propuesta como punto de partida para generar debate y diálogo intercultural para el consenso y el entendimiento del Cultivo que se pretende realizar, poniéndose de acuerdo y establecer criterios que parten de los planes de vida de los pueblos, que buscan responder a una necesidad, demanda o de igual manera fortalecer ciertos espacios que la comunidad y organización determine 
y consense con el investigador o investigadora. Todo ello parte del consentimiento previo, libre e informado como un aspecto fundamental y esencial. Consiste en el consenso comunitario, es decir, entre todos los involucrados en el proceso mediante los consentimientos, previos, libres e informados, definiendo el patrimonio de saberes y conocimientos, así lo describe el CCRISAC (p. 31).

\section{Segundo momento: “Análisis del contexto"}

Se inicia de una valoración e interpretación contextual sobre el estado del arte tomando en cuenta lo que se pretende cultivar. Asegura partir de un análisis crítico y propositivo sobre lo que se busca y lo que se quiere. Este se construye con base a las realidades y particularidades que refleja el posible problema a lo que se pretende responder. En este espacio técnicamente se reflejan los elementos integrales de la idea de investigación (p. 31).

\section{Tercer momento: Armonía entre saberes}

Permite armonizar los conocimientos occidentales con los tradicionales, asegura una relación y formulación de conceptos desde la cosmovisión de los pueblos indígenas y afrodescendientes, pero además desde el planteamiento científico en el marco de la revisión documental que se complementa con el compartir de la palabra de fuentes vivas, que ofrecen una mayor riqueza en ese diálogo inter epistémico que se relaciona con el conocimiento endógeno con el exógeno. Este tercer momento garantiza una conjugación amigable para dilucidar los diferentes planteamientos, experiencias y vivencias, dando así mayor rigurosidad y fortaleza al estudio. Y permite esa relación intercultural a través del intercambio de conocimientos (p. 31).

\section{Cuarto momento: Diseño de los caminos: Investigación Acción Intercultural}

Este momento es fundamental porque consiste en determinar la manera en que se desarrollan los procesos de Recreación de Conocimientos Saberes y Prácticas, es decir, la investigación acción intercultural; uno de los elementos fundamentales que consiste las acciones que determinar la convivencia, el diálogo, la interculturalidad, le correlación entre los actores como piezas fundamentales de la colectividad.

Este espacio se materializa mediante la convivencia y las vivencias que se determinan junto a las familias sujetos de estudio, (Calderón; 2018). Pero, además aporta directamente desde su construcción a un fortalecimiento donde la comunidad participa para reflexionar sobre situaciones y problemas que enfrentan (Velásquez; 2018). 


\section{Quinto momento: Convivencia y construcción comunitaria de conocimientos saberes y prácticas}

Garantiza el convivir, el intercambio, la relación directa de tu a tu desde el entorno comunitario, que garantiza vivenciar la aplicación de las técnicas metodológicas diseñadas para cultivar y crear el conocimiento con y para la comunidad. Iniciativa que vuelve única esta propuesta metodológica porque se materializar desde el Bien Hacer. Desde su implementación se visualiza el Diálogo de Saberes y Haceres, sí como el diálogo intercultural. Es la convivencia con el entorno comunitario (RUIICAY; 2018, p. 32).

\section{Sexto momento: Diálogo de saberes y haceres}

El Diálogo de Saberes y Haceres, es fundamental en el compartir la palabra, puesto que socializa los conocimientos desde las distintas perspectivas y vivencias que se materializan desde el seno de las comunidades. Este ejercicio garantiza el retroalimentar, valida, acoge, dialoga, debate y consolida para posteriormente consensuar. Este ejercicio se desarrolla desde la estructuración de la información hasta la socialización de los conocimientos y sabidurías (RUIICAY, 2018), y representa casi la parte final del diseño metodológico del Camino Creación y Recreación de Conocimientos Saberes y Prácticas.

\section{Séptimo momento: Plan de Acción}

De todo el caminar antes descrito finalmente concluye con un plan que se desprende de los resultados obtenidos a través de la investigación, que está listo para retornar a la comunidad y ser implementado desde el seno de su comunidad. Pero también es importante resaltar que antes que todo, primero se valida con la comunidad antes de ser presentado en cualquier instancia y esta hace las observaciones que estima conveniente desde la lógica planteada por el investigador. Aquí es donde finaliza esa etapa para iniciar su implementación. Esta es una de las principales características fundamentales del CCRISAC. Es el diseño y ejecución de un plan, proyecto o iniciativa diseñada conjuntamente con las comunidades (RUIICAY, 2018, p. 32).

\section{Conclusiones}

La comunicación iontercultural es un elemento indispensable en los procesos de Creación y Recreación de Conocimientos Saberes y Prácticas, que se materializa desde la relación y correlación entre los sujetos de estudio, es decir, las comunidades son el foco esencial que abren la mente al investigador para poder construir comunitaria e interculturalmente. Por ello, todo investigador e investigadora, debe de abrir su mente al ejercicio de una comunicación diferente, humana y que se hace visible desde el entendimiento respuetoso entre diferentes. 
Por lo tanto, las características que comparte el CCRISAC, son fundamentales para posicionar que el ejercicio de una investigación de sujeto a sujeto es posible, porque es una herramienta en construcción que permite la relación dialógica y horizontal que rompe paradigmas y crea espacios de consenso en armonía entre los distintos saberes.

Es necesaria profundizar y compartir los elementos que la hacen propia para que los investigadores e investigadoras se atrevan a incursionar en esta nueva propuesta que se fundamenta desde el pensamiento indígena, afrodescendiente de Abya Yala y que se materializa desde el seno de las comunidades.

El CCRISAC y la Comunicación Intercultural se conjungan de manera eficazy pertinente para la implementación de una metodología de investigación propia que aegura el involucramiento de los sujetos de manera colectiva y permanente. Esto le da un toque muy especial y particular porque materializa la interculturalidad desde la relación durante todo el proceso constructivo y luego en la debida implementación de los resultados.

\section{Agradecimiento}

Esta publicación obtuvo el financiamiento de: El Fondo de Asistencia Internacional de los Estudiantes y Académicos Noruegos, (SAIH).

\section{Lista de referencias}

Asunción-Lande, N. (1986). Comunicación intercultural. La comunicación humana ciencia social. México DF: Mc-Graw-Hill, 177-198.

Alsina, M. (1997). Elementos para una comunicación intercultural. Revista CIDOB D’Afers Internacionals, (36), 11-21. Retrieved from http://www.jstor.org/ stable/40550303

Alsina, M. R. (1999). La comunicación intercultural (Vol. 22). Anthropos Editorial.

Olabuénaga, J. I. R. (1999). Metodología de la investigación cualitativa. Universidad de Deusto. Bilbao.

Charris Benedetti, J. P. (2014). El consentimiento libre, previo e informado como garantía de transparencia en la administración pública. Revista derecho del estado (33).

Hooker Blandford, A. (2017). El papel de la comunicación en procesos de interculturalidad. URACCAN Al Día, 11(2), 14-17. Recuperado a partir de http://revistas. uraccan.edu.ni/index.php/uraccanaldia/article/view/414 
RUIICAY (2018). Cultivo y Crianza de Sabidurías y Conocimientos. URACCAN. Managua.

Montoya Ortega, Y. (2017). La interculturalidad y la comunicación intercultural: consolidando el Buen Vivir de los Pueblos. Revista Universitaria Del Caribe,19(2), 31-35. DOI: http://dx.doi.org/10.5377/ruc.v19i2.646

Velásquez Reyes, J. (2018). Gestión de la información a través de las Tecnologías de la Información y las Comunicaciones para el fortalecimiento de la comunicación intercultural en URACCAN, 2018. (Tesis inédita de Maestría) Universidad de las Regiones Autónomas de la Costa Caribe Nicaragüense, URACCAN. Bluefields.

Calderón Rivera, N. (2018). Prácticas comunicacionales de las familias creoles en contextos multiétnicos del Triángulo Minero, Región Autónoma del Caribe Norte de Nicaragua. (Tesis inédita de Maestría) Universidad de las Regiones Autónomas de la Costa Caribe Nicaragüense, URACCAN. Bluefields.

Timmer, H. (2003). La chakana. De Kosmos Fluistert Zijn Namen.

URACCAN (2014). Plan Estratégico Participativo de la URACCAN (PEI-2015-2019). URACCAN. Regiones Autónomas de la Costa Caribe Nicaragüense. 